\title{
Editorial
}

\section{Hybrid vectors: a new generation of virus- based vectors designed to control the cellular fate of delivered genes}

Gene therapy can be defined simply as the delivery of specific nucleic acid sequences to host cells, which results in a clinically relevant phenotypic change. The promise of this technology is that mechanisms of disease can be altered at a more fundamental level than conventional forms of treatment, leading to new and more effective interventions. Successful gene therapy is dependent on several steps, including targeting to the desired tissue, efficient transduction of cells, and maintenance of transgene expression for a period of time that is clinically significant. The most efficient vehicles for gene delivery are vector systems based on animal viruses. These vectors are designed to utilize specific biological properties of viruses, such as cell receptors for entry, that have evolved over time in relationship with the host. Furthermore, viral genetics are relatively simple, well characterized and easily manipulated; features that facilitate the design of vectors with predictable biological properties. Several different viruses have been used as the basis for gene delivery vectors, but the success of these systems is the product of two broad goals: maintaining the biological advantages of viruses that have been selected by nature, while reducing viral determinants of cytopathic effect.

A major hurdle in gene therapy is stable transgene expression. Long-term transgene expression is important because repeated administration augments the host immune response, which leads to inhibition of gene delivery, modulation of gene expression and immunopathology. Furthermore, the physical introduction of vectors can be invasive and contribute to cytopathic effect. After successful delivery has occurred, expression of the introduced transgene can be further diminished by promoter inactivation, physical loss or degradation of vector sequences, cytotoxic effect of the vector on the host cell and immune response to viral and transgene products. Current strategies for attaining vector-driven longterm expression include the introduction of promoter or enhancer elements that control stable, cell- or tissue-specific expression. However, the fundamental goal of stable transduction is likely to be dependent on the maintenance of the introduced vector sequences in a physical state that can support long-term gene expression. Vectors that exist as nonreplicating extrachromosomal elements in the nucleus of transduced cells, such as those derived from herpes simplex virus type 1 (HSV-1) and adenovirus (Ad), do not support long-term expression. If the target tissue is composed of dividing cells, these vectors are not retained efficiently in the progeny. More importantly, in nondividing cells, a single administration of Ad- or HSV-1-based vectors results in transgene expression that decays over time. We hypothesize that the introduced vector sequences in nondividing cells persist, but assume a conformation that inactivates transcription. This may be analogous to the latent stage of the HSV-1 life cycle, in which the viral genome becomes methylated and histone-bound, and gene expression is essentially quiescent. Therefore, long-term transgene expression may be critically related to the physical state of the vector sequences, particularly in respect to access of the introduced regulatory regions to the transcriptional machinery of the cell.

A new generation of gene delivery vectors has been introduced which is designed to control the fate of delivered transgenes within the nucleus of transduced cells. Specific viral elements have been incorporated which maintain the input DNA in a physical state that can support expression and replication. This has been achieved by adding elements that either direct integration into the host genome, or allow the vector to replicate autonomously as an episomal element within cells. These vectors use elements from different viruses that specify the physical state of the introduced vector sequences and have been termed 'hybrid' vectors. Hybrid vectors are intended to combine advantageous properties of current vector systems including broad host ranges, large transgene capacity and high titers, with heterologous elements that confer genetic stability to the vector DNA for long periods. Specific vectors are described in Table 1.

The lentiviral vectors have become the prototype for delivery systems that specify the cellular fate of transgenes. ${ }^{1}$ Unlike other retroviruses, human immunodeficiency virus type 1 (HIV-1) efficiently targets to the nucleus of infected cells and encodes a viral integrase that inserts a provirus copy into the genome of nondividing cells. HIV-1, however, has a limited host range, low titers, and determinants of pathogenesis that limit its utility as a vector system. These problems have been circumvented by retaining advantageous elements of HIV-1, reducing the viral determinants of pathogenesis, and substituting the HIV envelope proteins with the vesicular stomatitis virus (VSV) G surface glycoprotein. The vector is produced using a three plasmid cotransfection protocol: (1) an HIV-1 packaging plasmid; (2) a vector plasmid containing the HIV-1 packaging signal and viral integrase, with a CMV promoter-driven transgene; and (3) a plasmid that expresses the VSV G surface glycoprotein. Important features of the HIV-1 parent virus are retained, 
Table 1 Hybrid vectors

\begin{tabular}{|c|c|c|c|c|}
\hline Vector/Author/Ref & Viral backbone & $\begin{array}{l}\text { Parent vector } \\
\text { features }\end{array}$ & $\begin{array}{l}\text { Heterologous } \\
\text { elements }\end{array}$ & Intent of design \\
\hline $\begin{array}{l}\text { Lentivirus } \\
\text { Naldini et } a l^{1,2}\end{array}$ & HIV-1 & $\begin{array}{l}\text { (1) HIV integrase } \\
\text { (2) HIV packaging }\end{array}$ & $\begin{array}{l}\text { VSV G surface } \\
\text { protein }\end{array}$ & $\begin{array}{l}\text { (1) Integration in dividing and nondividing cells } \\
\text { (2) Broad host range } \\
\text { (3) High titers after purification }\end{array}$ \\
\hline $\begin{array}{l}\text { HSV / AAV } \\
\text { Johnston et } a l^{7}\end{array}$ & HSV-1 amplicon & HSV-1 packaging & $\begin{array}{l}\text { (1) AAV Rep gene } \\
\text { (2) AAV inverted } \\
\text { terminal repeats } \\
\text { (ITRs) }\end{array}$ & $\begin{array}{l}\text { (1) Site specific integration in dividing and } \\
\text { nondividing cells } \\
\text { (2) Transgene amplification }\end{array}$ \\
\hline $\begin{array}{l}\text { Ad/AAV } \\
\text { Fisher } \text { et } a l^{8}\end{array}$ & $\begin{array}{l}\text { Adenovirus } \\
\text { (Ad) }\end{array}$ & Ad packaging & $\begin{array}{l}\text { (1) AAV ITRs } \\
\text { (2) AAV Rep gene }\end{array}$ & $\begin{array}{l}\text { (1) Site-specific integration } \\
\text { (2) Transgene amplification }\end{array}$ \\
\hline $\begin{array}{l}\mathrm{HSV} / \mathrm{EBV} \\
\text { Wang and } \operatorname{Vos}^{11}\end{array}$ & HSV-1 amplicon & HSV-1 packaging & $\begin{array}{l}\text { (1) EBV ori-P } \\
\text { (2) EBV EBNA } \\
\text { gene }\end{array}$ & $\begin{array}{l}\text { Episomal retention and replication in dividing } \\
\text { cells }\end{array}$ \\
\hline
\end{tabular}

aAA Rep gene is added as an additional component to the Ad/AAV hybrid by polylysine conjugation of an expression plasmid to the recombinant Ad virions.

such as the packaging signal, and genes that encode integrase and nuclear localization. Nonessential HIV genes such as env and the regulatory genes vif, vpr, vpu and nef, which are virulence factors, have been deleted from the vector plasmid. In contrast to the HIV-1 parent, VSV pseudotyped lentiviral vectors have a broad host range and stable physical properties that allow purification of high titrated stocks. This vector system has been tested extensively in the post-mitotic environment of the central nervous system and has demonstrated: (1) transduction of neurons in vivo; (2) sustained transgene expression in post-mitotic cells for months; and (3) lack of inflammatory response. ${ }^{2,3}$ The HIV-1 integrase function is a necessary requirement for long-term transgene expression in post-mitotic cells, supporting the hypothesis that the physical state of the introduced sequences is critical for gene therapy of post-mitotic tissues.

We have constructed an HSV-1 based hybrid amplicon that combines desirable features of both the parent virus and adeno-associated virus (AAV). Amplicon vectors are shuttle plasmids that contain the HSV-1 elements necessary for both viral DNA replication and packaging into virions. Amplicons containing one or more genes of interest can be packaged into HSV-1 virions either by replication defective HSV-1 helper virus ${ }^{4}$ or by the recently described helper virus-free method. ${ }^{5}$ This vector has been modified by the addition of the AAV rep gene and the AAV inverted terminal repeats (ITRs). AAV ITRs are cisacting elements that in the presence of AAV Rep mediate both amplification and subsequent integration of a desired gene into a specific site (AAVS1) in the human genome on chromosome 19q13.3. ${ }^{6}$ The HSV / AAV hybrid vector is designed to utilize the HSV-1 properties of entry and nuclear localization, and the AAV properties of transgene amplification and integration into a nonessential locus in nondividing human cells. An essential question is whether AAV elements function in the context of an HSV-1 amplicon vector. Amplification of ITR-flanked transgenes from the hybrid amplicon has been demonstrated by rescue experiments, in which the transgene cassette is packaged into AAV virions when cotransfected with plasmids bearing the AAV structural (cap) gene in the presence of either Ad or HSV-1 helper functions (F Smith, unpublished results). In dividing cells, a HSV/AAV hybrid vector containing the neomycin resistance gene directs the formation of stable colonies in human cells that grow under selection and remain stable for $>25$ passages (D Jacoby, unpublished results). Moreover, $30-40 \%$ of hybrid vector transducedcells form stable colonies, in contrast to essentially no colony formation mediated by traditional HSV-1 amplicon vectors, suggesting that the HSV / AAV vector is capable of integration into dividing human cells. The hybrid vector, in comparison with traditional HSV-1 amplicon vectors, has been shown to extend transgene expression in dividing human glioma cells, ${ }^{7}$ human and rodent hepatic cell lines, and nondividing cells in the rodent liver ${ }^{12}$ and rodent CNS in vivo (D Jacoby, unpublished results). This vector, when packaged in the helper virus-free system, shows minimal cytopathic effect in culture and in vivo, and constitutes a 'virtual' synthetic vector in which delivery of the transgene is mediated by HSV-1 virions in the absence of HSV-1 genes.

A hybrid vector of similar rationale was constructed by inserting an AAV ITR flanked marker gene into a parent adenovirus (Ad5) deleted in the E1a and E1b genes. The Ad/AAV hybrid vector did not include the AAV rep gene due to its inhibitory effect on the replication of adenovirus. Instead, a plasmid expressing the AAV Rep isoforms was conjugated to the virion surface by a poly-I lysine bridge, thereby bringing in the AAV rep gene as an extraviral component. ${ }^{8}$ The Ad/AAV hybrid vector supports transgene amplification, as demonstrated by AAV packaging, but this system has not yet been characterized for its ability to direct transgene integration or long-term gene expression.

Lentivirus vectors and hybrid vectors containing AAV components are designed to promote stable long-term expression by integration into the host genome of slowly dividing, quiescent or post-mitotic cells. Vectors that undergo recombination with the host genome have theoretical drawbacks, such as insertional damage of host genes, or integration of the transgene into a transcriptionally silent locus. Of note, lentivirus vectors have not 
been associated with tumor formation, viral reactivation or transgene silencing. HIV-1-directed integration does not occur with known site specificity, and is postulated to favor regions of open chromatin, which may select for areas of high gene expression. ${ }^{9}$ Integration by this mechanism may reduce the probability of recombination at a silent locus, but may increase the likelihood of insertional mutagenesis. The HSV/AAV and Ad/AAV vectors are designed to integrate in a specific region in the human genome which has not been associated with pathology. ${ }^{10}$ Despite a lower risk of insertional mutagenesis, the AAVS1 site presents two remaining problems: (1) analogous sites in animal models have not been fully characterized; and (2) integration at this site may not support stable gene expression. The principles governing safety and efficacy of gene therapy vectors designed to recombine with the host genome are yet to be explored.

An HSV/EBV hybrid vector has been constructed that utilizes the Epstein-Barr virus (EBV) ori-P element and the EBV nuclear antigen 1 (EBNA-1) gene to maintain the vector DNA as an autonomous extrachromosomal unit in dividing cells. ${ }^{11}$ The HSV /EBV hybrid amplicon containing an antibiotic selection gene allowed formation of colonies in dividing cell lines under selection, that were stable for at least 6 months in culture. Co-infection of these cells with HSV-1 helper virus resulted in efficient packaging of the HSV/EBV amplicon, with amplicon/ helper ratios of up to 15:1. The presence of hybrid amplicon sequences in every dividing cell apparently resulted in a significant reduction in the helper virus interference observed in conventional amplicon packaging. This vector system may have important applications for gene therapy of dividing cells, such as in vivo delivery of prodrug activating genes in tumors.

The formation of 'hybrid' viruses is not a new technology; it has been used as a genetic tool by virologists for many years. Generation of heterotypic viruses has had several important applications: (1) making 'pseudotyped' viruses, ie altering surface glycoproteins in order to change the host range specificity; (2) intertypic recombination between viral strains to map specific viral characteristics, such as neurotropism; (3) reassortment of segmented viruses to map viral genes; and (4) the generation of expression vectors by recombination of a foreign gene into a non-essential locus. For the specific applications of gene therapy, hybrid vectors utilize the efficient delivery of current virus-based systems, but are also designed to extend transgene expression by including genetic elements that control the fate of the introduced sequences in the host cell nucleus. Genetic stability and the resulting potential for stable expression of intro- duced transgenes appears to be the next important step in development of virus-based systems. Achieving this goal would hopefully promote the field from simple gene delivery to the realm of clinically relevant gene therapy.

DR Jacoby, C Fraefel and $\mathrm{XO}$ Breakefield Molecular Neurogenetics Unit Department of Neurology Massachusetts General Hospital and Neuroscience Program

Harvard Medical School

Boston, MA 02114, USA

\section{References}

1 Naldini $\mathrm{L}$ et al. In vivo gene delivery and stable transduction of nondividing cells by a lentiviral vector. Science 1996; 272: 263 267.

2 Naldini L et al. Efficient transfer, integration, and sustained long-term expression of the transgene in adult rat brains injected with a lentiviral vector. Proc Natl Acad Sci USA 1996; 93: 11382-11388.

3 Blomer $\mathrm{U}$ et al. Highly efficient and sustained gene transfer in adult neurons with a lentivirus vector. J Virol 1997; 71: 66416649.

4 Geller AI, Breakefield XO. Defective HSV-1 vector expresses Escherichia coli $\beta$-galactosidase in cultured peripheral neurons. Science 1988; 241: 1667-1669.

5 Fraefel $C$ et al. Helper virus-free transfer of herpes simplex virus type 1 plasmid vectors into neural cells. J Virol 1996; 70: 71907197.

6 Kotin RM, Linden RM, Berns KI. Characterization of a preferred site on human chromosome $19 \mathrm{q}$ for integration of adeno-associated virus DNA by non-homologous recombination. EMBO J 1992; 11: 5071-5078.

7 Johnston KM et al. HSV/AAV hybrid amplicon vectors extend transgene expression in human glioma cells. Hum Gene Ther 1997; 8: 359-370.

8 Fisher $\mathrm{KJ}$ et al. A novel adenovirus-adeno-associated virus hybrid vector that displays efficient rescue and delivery of the AAV genome. Hum Gene Ther 1996; 7: 2079-2087.

9 Swain A, Coffin JM. Mechanism of transduction by retroviruses. Science 1992; 255: 841-845.

10 Linden RM et al. Site-specific integration by adeno-associated virus. Proc Natl Acad Sci USA 1996; 93: 11288-11294.

11 Wang S, Vos J-M. A hybrid herpesvirus infectious vector based on Epstein-Barr virus and herpes simplex virus type 1 for gene transfer into human cells in vitro and in vivo. J Virol 1996; 70: 8422-8430.

\section{Reference added in proof}

12 Fraefel $\mathrm{C}$ et al. Gene transfer into hepatocytes mediated by helper virus-free HSV/AAV hybrid vectors. Mol Med (in press). 